\title{
ACOUSTIC SHAPE OPTIMIZATION BASED ON ISOGEOMETRIC BEM WITH ADJOINT VARIABLE METHOD
}

\author{
HAIBO CHEN $^{1}$, JIE WANG $^{1}$, CHANGJUN ZHEN $^{2} \&$ LEILEI CHEN ${ }^{3}$ \\ ${ }^{1}$ CAS Key Laboratory of Mechanical Behavior and Design of Materials, \\ University of Science and Technology of China, China \\ ${ }^{2}$ Institute of Sound and Vibration Research, Hefei University of Technology, China \\ ${ }^{3}$ College of Civil Engineering, Xinyang Normal University, China
}

\begin{abstract}
The isogeometric analysis (IGA) has been applied to the boundary element method (BEM), forming the IGA BEM. In this work, we introduce the IGA BEM to 2D acoustic shape optimization. The key treatment is the acoustic sensitivity analysis using the adjoint variable method (AVM). Compared with the direct differentiation method (DDM), the AVM is more suitable for problems with a large number of design variables. The gradient-based optimization solver is applied in order to update the design variables during the optimization iteration process and the Burton-Miller method is adopted to conquer the fictitious eigen-frequency problem in solving exterior acoustic problems. An example of scattering by an infinite rigid cylinder is presented to demonstrate the improved accuracy of IGA BEM. Then we verify the efficiency of the developed sensitivity algorithms through the example and demonstrate their potential in solving large-scale engineering problems. Finally, an optimization example is provided to validate the proposed optimization procedure. Numerical tests show that the optimal results are strongly frequency dependent.

Keywords: isogeometric analysis, boundary element method, acoustics sensitivity analysis, shape optimization.
\end{abstract}

\section{INTRODUCTION}

Reducing sound emission of structures is of great concern in engineering problems, and shape optimization has been proven to be an effective approach [1]. Compared with the conventional BEM, the IGA BEM offers a higher accuracy in solving acoustic problems [2], as the geometry is accurately described by using the control points. Since the geometry can be easily changed by changing the control points, it is very convenient to carry out the shape optimization by the IGA BEM. However, gradient-based optimization solvers usually require gradient information, which leads to an additional step, i.e. the sensitivity analysis. This yields extra computational efforts and thus makes the efficient analysis techniques indispensable.

In recent years, several works about sensitivity analysis and optimization have been made for acoustic problems. Zheng et al. [3]-[5] has made many efforts in sensitivity analysis methods by BEM in 3D acoustics. Chen et al. [6]-[9] also investigated sensitivity analysis with single design parameter by FEM-BEM both in 2D and 3D acoustics. Liu et al. [2] has carried out the shape optimization of noise barrier by IGA BEM with the differentiation method (DDM) in sensitivity analysis. However, these aforementioned works mainly use the DDM with conventional BEM for the sensitivity analysis. But in many engineering problems, multi design variables should be adopted to describe the shape accurately, which yields more computational efforts in the sensitivity analysis. To this end, this paper presents a shape optimization procedure based on the IGA BEM in 2D acoustics. The formulations of the design sensitivity analysis by using the adjoint variable method 
$(\mathrm{AVM})$ are derived. The gradient-based optimization solver is applied to update the design variables for minimizing the sound pressure at observation points.

\section{IGA BEM IN 2D ACOUSTICS}

For the IGA BEM in 2D acoustics, a series of efforts have been made by Chen et al. [1] and Liu et al [2]. To conquer the fictitious eigen-frequency problem, we use the conventional boundary integral equation (CBIE) and the hypersingular BIE (HBIE) as follows:

$$
\begin{aligned}
& \text { CBIE: } c(x) p(x)+\int F(x, y) p(y) d S(y)=\int G(x, y) q(y) d S(y), \\
& H B I E: c(x) q(x)+\int F^{1}(x, y) p(y) d S(y)=\int G^{1}(x, y) q(y) d S(y),
\end{aligned}
$$

where $G(x, y) 、 F(x, y) 、 G^{1}(x, y) 、 F^{1}(x, y)$ are the kernel functions. According to the definition of IGA, we have the NURBS interpolation [2] as follows:

$$
\begin{aligned}
& x(\xi)=\sum_{i=1}^{n} R_{i, p_{g}}^{g}(\xi) X_{i} \\
& p(\xi)=\sum_{i=1}^{n_{f}} R_{i, p_{f}}^{f}(\xi) p_{i} \\
& q(\xi)=\sum_{i=1}^{n_{f}} R_{i, p_{f}}^{f}(\xi) q_{i} .
\end{aligned}
$$

Substituting eqn (3) into the CBIE and HBIE, the discretized isogeometric BIEs can be obtained as:

$$
\begin{gathered}
c\left(\boldsymbol{x}\left(\hat{\xi}_{i}\right)\right) \sum_{j=1}^{n_{f}} R_{j, p_{f}}^{f}\left(\hat{\xi}_{i}\right) p_{j}+\sum_{e=1}^{N_{e}} \sum_{j=1}^{n_{f}}\left[\int_{\xi_{e}}^{\xi_{e+1}} F\left(\boldsymbol{x}\left(\hat{\xi}_{i}\right), \boldsymbol{y}(\xi)\right) R_{j, p_{f}}^{f}(\xi) J(\xi) d \xi\right] p_{j} \\
=\sum_{e=1}^{N_{e}} \sum_{j=1}^{n_{f}}\left[\int_{\xi_{e}}^{\xi_{e+1}} G\left(\boldsymbol{x}\left(\hat{\xi}_{i}\right), \boldsymbol{y}(\xi)\right) R_{j, p_{f}}^{f}(\xi) J(\xi) d \xi\right] q_{j} \quad i=1,2, \cdots, n_{f}, \\
c\left(\boldsymbol{x}\left(\hat{\xi}_{i}\right)\right) \sum_{j=1}^{n_{f}} R_{j, p_{f}}^{f}\left(\hat{\xi}_{i}\right) q_{j}+\sum_{e=1}^{N_{e}} \sum_{j=1}^{n_{f}}\left[\int_{\xi_{e}}^{\xi_{e+1}} F^{1}\left(\boldsymbol{x}\left(\hat{\xi}_{i}\right), \boldsymbol{y}(\xi)\right) R_{j, p_{f}}^{f}(\xi) J(\xi) d \xi\right] p_{j} \\
=\sum_{e=1}^{N_{e}} \sum_{j=1}^{n_{f}}\left[\int_{\xi_{e}}^{\xi_{e+1}} G^{1}\left(\boldsymbol{x}\left(\hat{\xi}_{i}\right), \boldsymbol{y}(\xi)\right) R_{j, p_{f}}^{f}(\xi) J(\xi) d \xi\right] q_{j} \quad i=1,2, \cdots, n_{f},
\end{gathered}
$$

where $N_{e}$ is the number of NURBS elements, and $\left[\xi_{e}, \xi_{e+1}\right]$ is a NURBS element.

According to the Burton-Miller method, giving coefficient $\alpha$ as follows:

$$
\begin{aligned}
& k<1, \alpha=i \\
& k \geq 1, \alpha=\frac{i}{k},
\end{aligned}
$$

where $k$ is the wave number. Then we have the following equation:

$$
C B I E+\alpha H B I E=0 .
$$

Performing linear combination to eqn (7), the following linear algebraic equations in matrix form are obtained: 


$$
\boldsymbol{H} p=\boldsymbol{G} q
$$

Moving all the unknowns of eqn (8) to the left-hand side and all the known values to the right-hand side, the linear equations are given by:

$$
\boldsymbol{A} X=b .
$$

Finally, we obtain the unknowns by solving eqn (9).

\section{ACOUSTIC SENSITIVITY ANALYSIS WITH ADJOINT VARIABLE METHOD}

Sensitivity analysis plays a great role in the shape optimization. Here the coordinates of control points in IGA can be chosen as the design variables. Some works about sensitivity analysis using the DDM [1]-[3], [5]-[7] have been developed. According to the DDM, eqns (1) and (2) can be differentiated with respect to the design variables as follows:

$$
\begin{gathered}
c(x) \dot{p}(x)+\int F(x, y) \dot{p}(y) d S(y)+\int \dot{F}(x, y) p(y) d S(y)+\int F(x, y) p(y) d \dot{S}(y) \\
=\int G(x, y) \dot{q}(y) d S(y)+\int \dot{G}(x, y) q(y) d S(y)+\int G(x, y) q(y) d \dot{S}(y), \\
c(x) \dot{q}(x)+\int F^{1}(x, y) \dot{p}(y) d S(y)+\int \dot{F}^{1}(x, y) p(y) d S(y)+\int F^{1}(x, y) p(y) d \dot{S}(y) \\
=\int G^{1}(x, y) \dot{q}(y) d S(y)+\int \dot{G}^{1}(x, y) q(y) d S(y)+\int G^{1}(x, y) q(y) d \dot{S}(y),
\end{gathered}
$$

where the upper $\operatorname{dot}\left({ }^{\circ}\right)$ denotes the differentiation with respect to the design variables.

Substituting eqn (3) into eqns (10) and (11), and still applying the Burton-Miller method, we get the linear algebraic equations in matrix form as:

$$
\dot{\boldsymbol{H}} p+\boldsymbol{H} \dot{p}=\dot{\boldsymbol{G}} q+\boldsymbol{G} \dot{q}
$$

where the matrix $\boldsymbol{H} 、 \boldsymbol{G}$ have been calculated in the IGA BEM. By swapping the unknowns, we can obtain the unknown sensitivity values by the following equation:

$$
\boldsymbol{A} \dot{\psi}=\boldsymbol{B} \dot{\phi}-\dot{\boldsymbol{H}} p+\dot{\boldsymbol{G}} q
$$

where $\dot{\psi}$ is the unknown sensitivity values. If the sound pressures at the field points are included in the objective function, the sensitivity values are given by:

$$
\dot{p}_{\text {inner }}(x)=\{u\}^{T} \dot{\psi}+\{k\}^{T} \dot{\phi}+\{\dot{e}\}^{T} q-\{\dot{b}\}^{T} p .
$$

However, if the objective function in optimization is only related to the field points, AVM could be used. According to eqn (13), we have:

$$
\dot{\psi}=\boldsymbol{A}^{-1}\{\boldsymbol{B} \dot{\phi}-\dot{\boldsymbol{H}} p+\dot{\boldsymbol{G}} q\} .
$$


Introducing the adjoint equations as follows:

$$
\begin{gathered}
\boldsymbol{A}^{T} \eta=u, \\
\{\eta\}^{T}=\{u\}^{T} \boldsymbol{A}^{-1} .
\end{gathered}
$$

In addition, by substituting eqns (15) and (16) into eqn (14), the new formulation of field points' sensitivities can be obtained as

$$
\dot{p}_{\text {inner }}(x)=\{\eta\}^{T}\{\boldsymbol{B} \dot{\phi}-\dot{\boldsymbol{H}} p+\dot{\boldsymbol{G}} q\}+\{k\}^{T} \dot{\boldsymbol{\phi}}+\{\dot{e}\}^{T} q-\{\dot{b}\}^{T} p
$$

As we can see, eqn (16) is independent with all of the design variables. Hence, we only need to compute it once even if there exist a larger number of design variables. This will significantly improve the efficiency of sensitivity analysis, which will play a great role in the following optimization process.

\section{SHAPE OPTIMIZATION PROCESS}

After the sensitivity analysis by the AVM, we choose the method of moving asymptotes (MMA) as the optimization solver. The minimization of sound pressure on a specified reference plane is set as the design objective. The optimization model can be formulated as follows:

$$
\begin{gathered}
\min : \Pi(\boldsymbol{x})=\bar{p}_{s}(\boldsymbol{x}) p_{s}(\boldsymbol{x}) \\
\text { s.t.: } A(\boldsymbol{x}) \leq A_{0} \\
\text { bound: } x_{\text {mini }} \leq x_{i} \leq x_{\text {maxi }}, i=1,2, \cdots, n_{d} \\
\text { iteration: }\left|\frac{\Pi^{m+1}-\Pi^{m}}{\Pi^{m}}\right|<\tau,
\end{gathered}
$$

where $\boldsymbol{x}$ is a vector of control points' coordinates $x_{i}\left(i=1,2, \cdots, n_{d}\right)$ that are set as design variables; $\Pi(\boldsymbol{x})$ is the objective function; $p_{s}(\boldsymbol{x})$ denotes the sound pressure of field points which located on the reference plane, and $\bar{p}_{S}(\boldsymbol{x})$ denotes the conjugate transpose of $p_{s}(\boldsymbol{x})$. $A_{0}$ is the initial area of structures, which is called constraint. $x_{\text {mini }}$ and $x_{\text {maxi }}$ are the lower and upper bounds for every design variable $x_{i} ; \tau$ is the given value which decide the iteration termination.

\section{NUMERICAL EXAMPLES}

To validate the proposed approach, some numerical examples are carried out. We consider an infinite rigid cylinder excited by plane wave (wave speed $\mathrm{c}=340 \mathrm{~m} / \mathrm{s}$ ) as shown in Fig. 1.

Firstly we compare the IGA BEM with the conventional BEM with different Lagrange basis functions. As Fig. 2 shows, IGA BEM achieves a higher accuracy than the constant, discontinuous linear and discontinuous quadratic elements (DBE21, DBE22 and DBE33, respectively). Then Fig. 3 shows the correctness of the sensitivity analysis. We compare the two analytical methods, i.e., the DDM and AVM, with finite difference method (FDM), and all of them give the same results. Finally we carry out the shape optimization of a rectangle structure shown in Fig. 4. A unit monopole is located at the point $(0,1)$. The thickness of the structure is set as $0.2 \mathrm{~m}$. Seventy-seven observation points are evenly distributed on the reference plane. The average sound pressure at these points is used as the objective function, as shown in eqn (18). 
To demonstrate the advantage of AVM, we choose 19 design variables (from control point $\mathrm{P}_{2}$ to $\mathrm{P}_{20}$ ) as shown in Fig. 5. The lower and upper bounds of these control points' horizontal coordinates are 4.9 and 5.1, respectively. The parameter $\tau$ is set as $10^{-4}$. And the constraint is that the structure should not go beyond the initial area.

For excitation frequencies $100 \mathrm{~Hz}, 400 \mathrm{~Hz}, 700 \mathrm{~Hz}$ and $1000 \mathrm{~Hz}$ are considered, and the optimized shapes are illustrated in Fig. 6. From the figure, it can be seen that different frequency produce different shape which indicates the optimized shape is strongly frequency dependent. Further, the optimized shape tends to be more complex as frequency increases, which will bring more peaks and valleys.

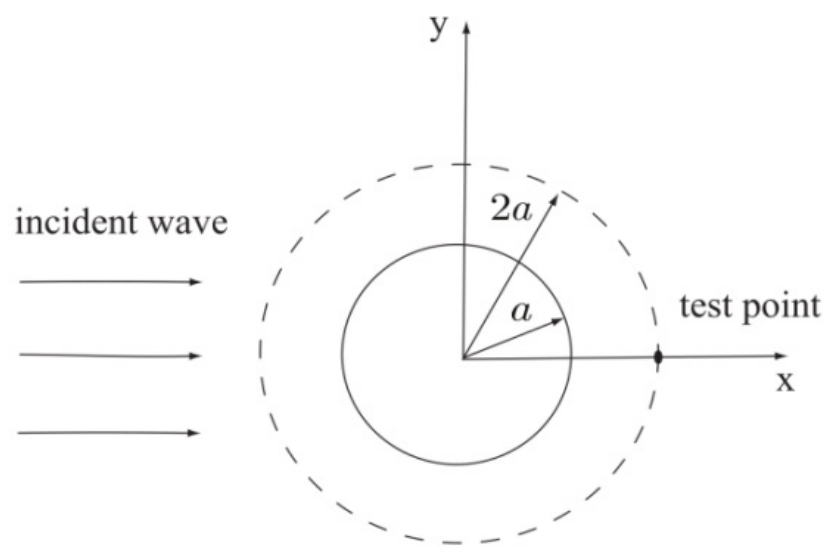

Figure 1: Scattering from an infinite rigid cylinder.

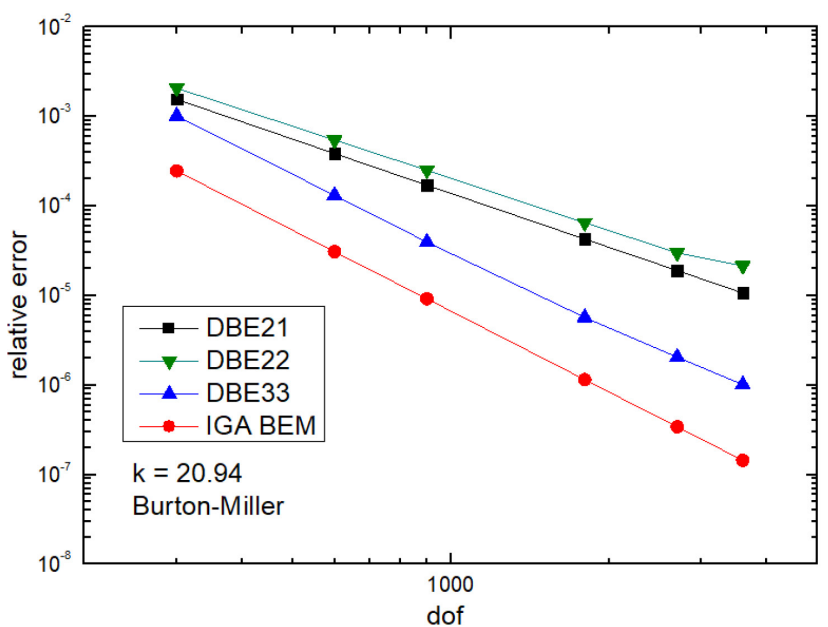

Figure 2: Comparison of the relative error of discontinued BEM and IGA BEM. 


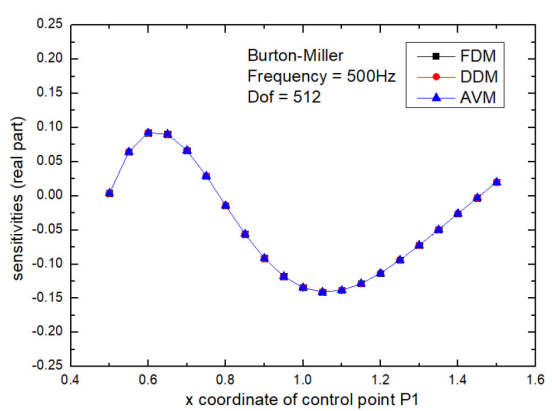

(a)

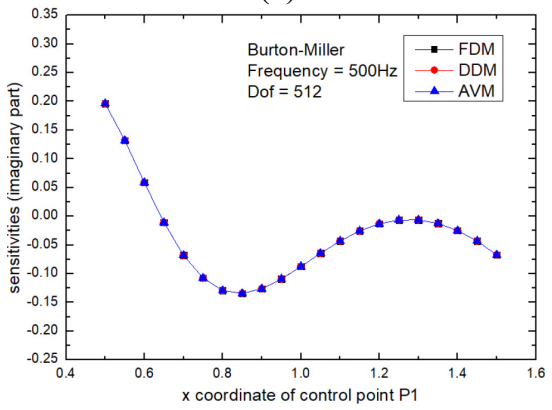

(b)

Figure 3: Sound pressure sensitivities at the field point $(2,0)$ with respect to the horizontal coordinate of control point $\mathrm{P}_{1}(1,0)$. (a) Real part; and (b) Imaginary part.

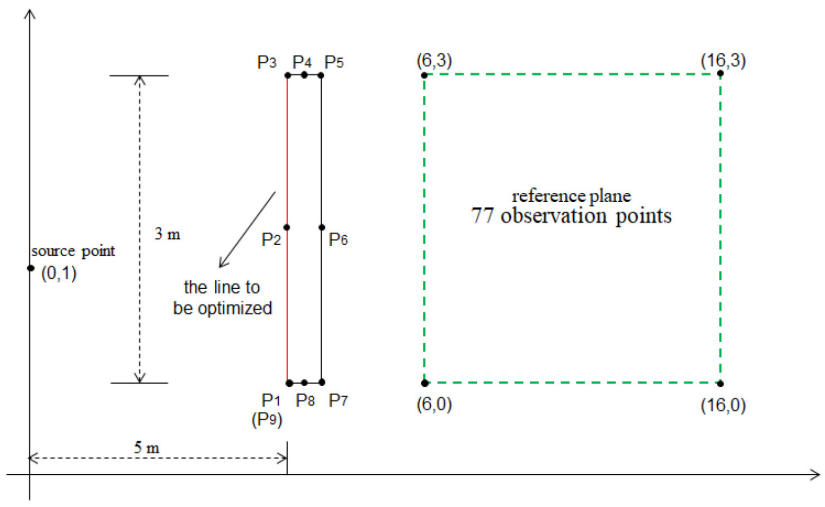

Figure 4: Initial rectangle structure.

The reduction of sound pressure level (SPL) is presented in Fig. 7. From the figure, we can see that the SPL values at $100 \mathrm{~Hz}$ reduce less than those at higher frequencies, which means that the optimization achieves better SPL reduction at higher frequencies. This can be explained that the higher the frequency, the shorter the wavelength, the less the diffraction by structures. 


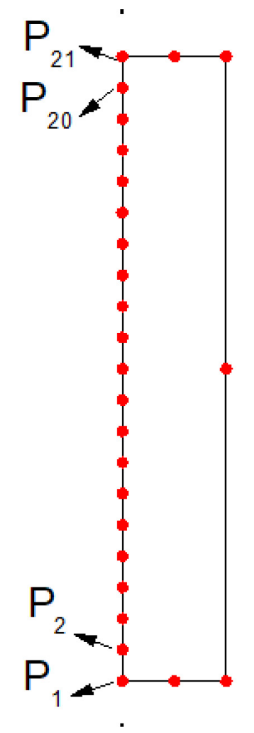

Figure 5: Design variables.

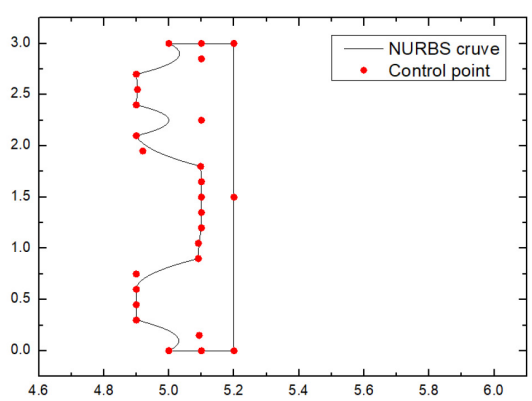

(a)

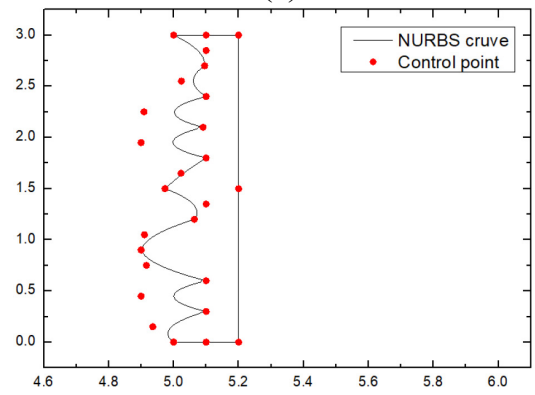

(c)

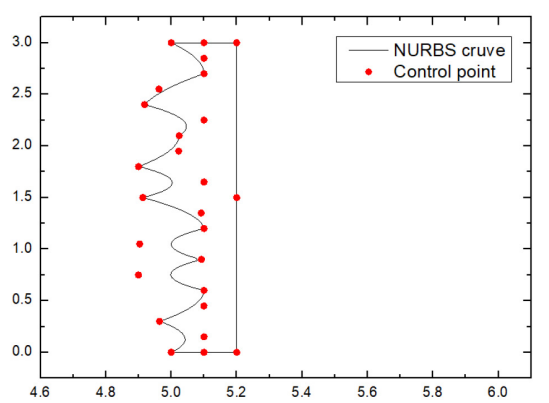

(b)

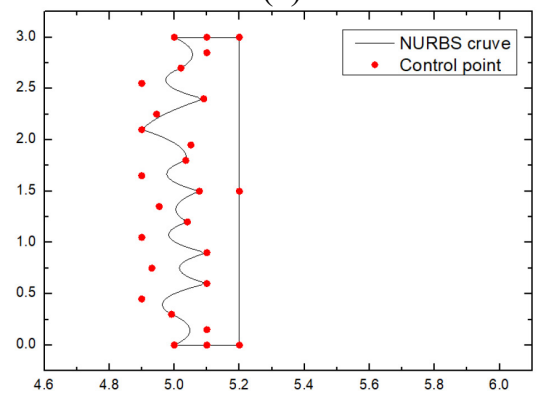

(d)

Figure 6: Shape optimization results with 19 design variables at different frequencies. (a) Frequency $=100 \mathrm{~Hz}$; (b) Frequency $=400 \mathrm{~Hz}$; (c) Frequency $=700 \mathrm{~Hz}$; and (d) Frequency $=1000 \mathrm{~Hz}$. 


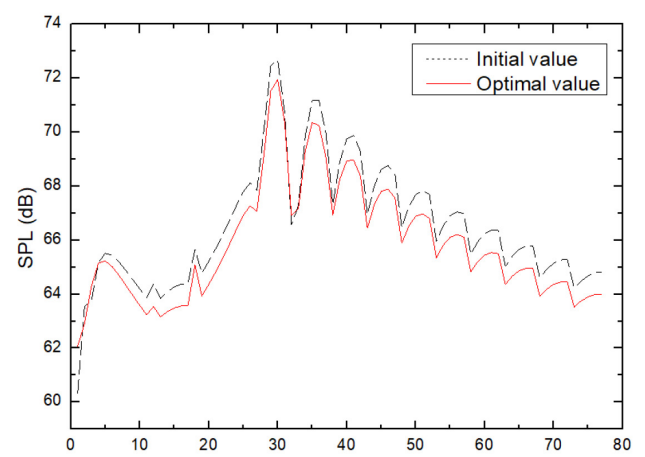

(a)

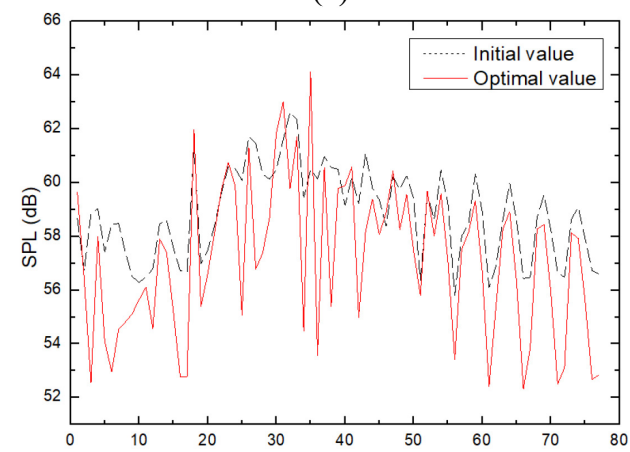

(c)

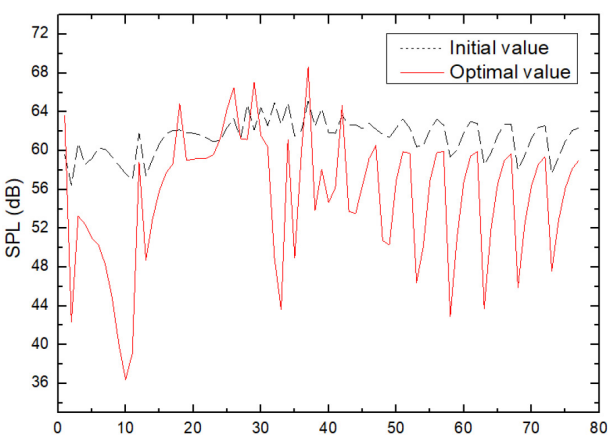

(b)

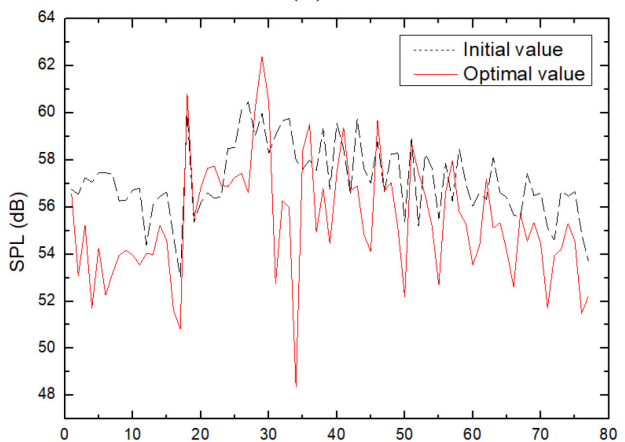

(d)

Figure 7: Reduction of SPL with 19 design variables at different frequencies. (a) Frequency $=100 \mathrm{~Hz}$; (b) Frequency $=400 \mathrm{~Hz}$; (c) Frequency $=700 \mathrm{~Hz}$; and (d) Frequency $=1000 \mathrm{~Hz}$.

\section{CONCLUSIONS}

This work presents a shape optimization approach based on the IGA BEM in 2D acoustics. The key treatment is the use of adjoint variable method for the design sensitivity analysis. Numerical tests show the validity of the developed approach. Furthermore, numerical simulation shows that IGA BEM is able to solve acoustic problems with high accuracy, and the proposed sensitivity analysis method is able to calculate the sensitivity values efficiently. The shape optimization example shows that the optimal results are strongly frequency dependent. The proposed optimization approach has the potential in application for engineering problems.

\section{ACKNOWLEDGEMENTS}

This work is financially supported by the National Natural Science Foundation of China (NSFC) under Grant Nos. 11772322 and 11872168, and the Strategic Priority Research Program of the Chinese Academy of Sciences under Grant No.XDB22040502. 


\section{REFERENCES}

[1] Chen, L.L., Liu, C., Zhao, W.C. \& Liu, L.C., An isogeometric approach of two dimensional acoustic design sensitivity analysis and topology optimization analysis for absorbing material distribution. Comput. Methods Appl. Mech. Engrg., 336(3), pp. 507-532, 2018.

[2] Liu, C., Chen, L.L., Zhao, W.C. \& Chen, H.B., Shape optimization of sound barrier using an isogeometric fast multipole boundary element method in two dimensions. Engineering Analysis with Boundary Elements, 85(9), pp. 142-157, 2017.

[3] Zheng, C.J., Matsumoto, T., Takahashi, T. \& Chen, H.B., Explicit evaluation of hypersingular boundary integral equations for acoustic sensitivity analysis based on direct differentiation method. Engineering Analysis with Boundary Elements, 35(11), pp. 1225-1235, 2011.

[4] Zheng, C.J., Chen, H.B., Matsumoto, T. \& Takahashi, T., 3D Acoustic shape sensitivity analysis using fast multipole boundary element method. International Journal of Computational Methods, 9(1), pp. 1240004, 2012.

[5] Zheng, C.J., Matsumoto, T., Takahashi, T. \& Chen, H.B., A wideband fast multipole boundary element method for three dimensional acoustic shape sensitivity analysis based on direct differentiation method. Engineering Analysis with Boundary Elements, 36(3), pp. 361-371, 2012.

[6] Chen, L.L., Zheng, C.J. \& Chen, H.B., A wideband FMBEM for 2D acoustic design sensitivity analysis based on direct differentiation method. Computation Mechanics, 52(3), pp. 631-648, 2013.

[7] Chen, L.L., Chen, H.B. \& Marburg, S., Structural-acoustic design sensitivity analysis based on direct differentiation method with different element types. Computer Modeling in Engineering \& Science, 107(3), pp. 249-276, 2015.

[8] Chen, L., Chen, H., Zheng, C. \& Marburg, S., Structural-acoustic sensitivity analysis of radiated sound power using a finite element / discontinuous fast multipole boundary element scheme. International Journal for Numerical Methods in Fluids, 82(12), pp. 858-878, 2016.

[9] Chen, L.L., Liu, L.C., Zhao, W.C. \& Chen, H.B., 2D Acoustic design sensitivity analysis based on adjoint variable method using different types of boundary elements. Acoustics Australia, 44(2), pp. 343-357, 2016.

[10] Zheng, C.J., Bi, C.X., Zhang, C.Z., Zhang, Y.B. \& Chen, H.B., Fictitious eigenfrequencies in the BEM for interior acoustic problems. Engineering Analysis with Boundary Elements, 104(3), pp. 170-182, 2019. 